\title{
Pseudo-Differential Projections and the Topology of Certain Spaces of Elliptic Boundary Value Problems
}

\author{
B. Booss-Bavnbek ${ }^{1}$ and K. P. Wojciechowski ${ }^{2}$ \\ 1 IMUFA, Roskilde Universitetscenter, DK-4000 Roskilde, Denmark \\ 2 Department of Mathematical Science, IUPUI, Indianapolis, IN 46223, USA
}

\begin{abstract}
We calculate the homotopy groups of the space of elliptic boundary value problems for an elliptic differential operator $A$ of a first order and of the space of elliptic self-adjoint boundary value problems when $A$ is a formally self-adjoint. In particular we show that the spectral flow of an $S^{1}$ family of self-adjoint elliptic boundary value problems is well defined. This provides some information on spectral properties along the lines of the Vafa-Witten approach to spectral inequalities.
\end{abstract}

\section{The Notion of an Elliptic Boundary Value Problem}

Let $X$ be a smooth compact manifold with boundary $Y$. Let $E$ and $F$ be smooth complex vector bundles over $X$. For simplicity, we consider $A: C^{\infty}(X ; E) \rightarrow C^{\infty}(X ; F)$ an elliptic differential operator over $X$ of first order. We discuss the results for a larger class of operators at the end of the paper. Now let us fix a Riemannian metric on $X$ and Hermitian structures on $E$ and $F$. Then in a fixed collar neighbourhood $N \cong I \times Y$ of $Y \cong 0 \times Y$ the operator $A$ takes the form

$$
A(t, y)=G(t, y)\left(\partial_{t}+B_{t}(y)\right)
$$

where $G(t, y):\left.\left.E\right|_{Y} \rightarrow F\right|_{Y}$ is a bundle isomorphism for fixed $t ; \partial_{t}$ is the normal derivative; and $B_{t}: C^{\infty}\left(Y ;\left.E\right|_{Y}\right) \rightarrow C^{\infty}\left(Y ;\left.E\right|_{Y}\right)$ is an elliptic differential operator of first order on $Y$ such that the principal symbol $b_{t}(y, \zeta)$ of $B_{t}$ has no purely imaginary eigenvalues. We will assume that $G(t, y)$ is unitary.

It is well known that the orthogonal (with respect to $L^{2}\left(Y ;\left.E\right|_{Y}\right)$ ) projection

$$
P(A): C^{\infty}\left(Y ;\left.E\right|_{Y}\right) \rightarrow H(A)
$$

of $C^{\infty}\left(Y ;\left.E\right|_{Y}\right)$ onto $H(A)=\left\{\left.u\right|_{Y} \mid u \in C^{\infty}(X ; E)\right.$ and $A u=0$ in $\left.X \backslash Y\right\}$ is a pseudodifferential operator of order zero. It is called the Calderón projector of $A$. At each point $(y, \zeta)$ of the cotangent sphere bundle $S Y$ the principal symbol of $P(A)$ is the orthogonal projection $p^{+}(y, \zeta): E_{y} \rightarrow E_{y}$ onto the direct sum $E_{y, \zeta}^{+}$of the eigenspaces of the symbol $b_{0}(y, \zeta)$ corresponding to the eigenvalues with positive real part.

Now let $V$ be a Hermitian bundle over $Y$ and let $R: C^{\infty}\left(Y ;\left.E\right|_{Y}\right) \rightarrow C^{\infty}(Y ; V)$ be 
a pseudo-differential operator. Then we have various expressions of a boundary value problem, namely the pair

$$
(A, R): C^{\infty}(X ; E) \rightarrow C^{\infty}(X ; F) \oplus \operatorname{ran}(R),
$$

the realization of $A$

$$
A_{r}: \operatorname{dom}\left(A_{R}\right) \stackrel{A}{\rightarrow} L^{2}(X ; F)
$$

as an unbounded operator in $L^{2}(X ; E)$ with

$$
\operatorname{dom}\left(A_{R}\right):=\left\{u \in L^{2}(X ; E) \mid A u \in L^{2}(X ; F) \text { and } R\left(\left.u\right|_{Y}\right)=0\right\},
$$

and the boundary integral

$$
R P(A): H(A) \rightarrow \operatorname{ran}(R) .
$$

It has been shown in Seeley [11] that we get well defined operators with suitable regularity and finite index under the following assumptions:

(a) the range is $R$ is closed in a suitable $L^{2}$ space, and

(b) the principal symbol $r$ of $R$ maps the range $E^{+}$of $p^{+}$isomorphically onto the range of $r$.

Remark. This notion includes the local condition (of Shapiro-Lopatinsky type) and the global condition (of Atiyah-Patodi-Singer type) as well. We get the local condition when $R: C^{\infty}\left(Y ;\left.E\right|_{Y}\right) \rightarrow C^{\infty}(Y ; V)$ is a pseudo-differential operator such that $r: R^{+} \rightarrow \pi^{*}(V)$ is an isomorphism where $\pi: S Y \rightarrow Y$ denotes the natural projection.

Note that here we also admit operators $R: C^{\infty}\left(Y ;\left.E\right|_{Y}\right) \rightarrow C^{\infty}\left(Y ;\left.E\right|_{Y}\right)$. In this case the cokernel of $R$ is infinite dimensional; but the point is that only the kernel of $R$ is important. Thus, as was explained precisely in Seeley [11], we may restrict ourselves to boundary conditions which are pseudo-differential projections. This leads us to the following definition:

1 Definition. We shall say that a pseudo-differential operator $R: C^{\infty}\left(Y ;\left.E\right|_{Y}\right) \rightarrow$ $C^{\infty}\left(Y ;\left.E\right|_{Y}\right)$ defines a generalized Atiyah-Patodi-Singer elliptic boundary condition for $A$ if and only if

(a) $R^{2}=R$, and

(b) $r(y, \zeta)=p^{+}(y, \zeta)$ for all $y \in Y$ and $\zeta \in S Y_{y}$.

We denote the space of pseudo-differential projections defining generalized APS conditions for $A$ by $\operatorname{Ell}_{Y}(A)$. It carries the $L^{2}$ operator topology.

Remark. The space $\operatorname{Ell}_{Y}(A)$ does not contain all possible elliptic boundary conditions for $A$. Even so, it is in a certain sense generic, and one can find a close correspondence between $\operatorname{Ell}_{Y}(A)$ and the Grassmannians which are used in Pressley-Segal [10], Segal-Wilson [12] Witten [15].

The introduction of the space $\operatorname{Ell}_{Y}(A)$ permits one to reduce the topological analysis of the space of elliptic boundary value problems to just the boundary as shown in the next theorem, which is an easy consequence of Seeley's considerations in $[11]$. 
2 Theorem. The space $\mathrm{Ell}_{Y}(A)$ of generalized Atiyah-Patodi-Singer elliptic boundary conditions for $A$ is isomorphic to the space

$$
\begin{array}{r}
P \operatorname{diff}_{p^{+}}\left(\left.E\right|_{Y}\right)=\left\{P \mid P^{2}=P \text { is pseudo-differential of } 0\right. \text {-th order over } \\
\left.Y \text { in } E \text { with principal symbol } p=p^{+}\right\} .
\end{array}
$$

\section{Spaces of Pseudo-Differential Projections With the Same Principal Symbol}

The space $P \operatorname{diff}_{p^{+}}\left(\left.E\right|_{Y}\right)$, or for short, $P \operatorname{diff}_{p^{-}}$was introduced in [16] and its homotopy groups were determined in [18] (see also [17] for the calculations concerning the connected components of $\left.P \operatorname{diff}_{p^{+}}\right)$. In particular we know that

$$
\pi_{i}\left(P \operatorname{diff}_{p^{+}}\right) \cong\left\{\begin{array}{ll}
\mathbf{Z} & \text { for } i \text { even } \\
0 & \text { for } i \text { odd }
\end{array},\right.
$$

which gives us the following result:

3 Theorem. Under the assumptions of Sect. 1 we have

$$
\pi_{i}\left(\mathrm{Ell}_{Y}(A)\right) \cong\left\{\begin{array}{ll}
\mathbf{Z} & \text { for } i \text { even } \\
0 & \text { for } i \text { odd }
\end{array} .\right.
$$

We present the following example which shows how Theorem 3 works. Let us assume that $X$ is an odd-dimensional compact manifold with boundary $Y$, and let us consider the Dirac operator $D: C^{\infty}(X ; S) \rightarrow C^{\infty}(X ; S)$ over $X$. It is a formally self-adjoint operator and it is of the form of Eq. (1), namely $D=G\left(\partial_{t}+B\right)$ over the collar when it is assumed that the metric is product near the boundary.

Now $G^{2}=-I d$ and $S$ decomposes over $Y$ into $\left.S\right|_{Y}=S_{+} \oplus S_{-}$. With respect to this decomposition we get

$$
D=\left(\begin{array}{cc}
i & 0 \\
0 & -i
\end{array}\right)\left(\partial_{t}+\left(\begin{array}{cc}
0 & B_{-}=B_{+}^{*} \\
B_{+} & 0
\end{array}\right)\right),
$$

where $B_{+}: C^{\infty}\left(Y ; S_{+}\right) \rightarrow C^{\infty}\left(Y ; S_{-}\right)$is the Dirac operator on $Y$. Let us observe that the formal self-adjointness of $D$ implies $G B=-B G$ (see below Sect. 3). This leads us to the following interesting observation:

Let $\phi_{\lambda}$ be an eigenfunction of $B, B \phi_{\lambda}=\lambda \phi_{\lambda}$ with $\lambda \neq 0$, then $G \phi_{\lambda}$ is an eigenfunction of $B$ with eigenvalue $-\lambda$. This means that the spectrum of $B$ is symmetric with respect to 0. Moreover, as shown in Palais [9; Chap. XVII], we have index $B_{+}=0$. This means that $\operatorname{dim} \operatorname{ker} B=\operatorname{dim} \operatorname{ker} B_{+}+\operatorname{dim} \operatorname{ker} B_{-}=2 \operatorname{dim} \operatorname{ker} B_{+}$.

Now let us consider the standard Atiyah-Patodi-Singer realization of $D$ i.e. $D_{\Pi I_{+}(B)}=\not D$ with the domain

$$
\operatorname{dom} D_{\Pi_{+}(B)}=\left\{u \in H^{1}(S) \mid \Pi_{+}(B)\left(\left.u\right|_{Y}\right)=0\right\},
$$

where $\Pi_{+}(B)$ denotes the projection of $L^{2}\left(\left.S\right|_{Y}\right)$ onto the subspace spanned by the eigenfunctions of $B$ corresponding to the non-negative eigenvalues. Now, from the results of Atiyah-Patodi-Singer [2] we know that $D_{\Pi_{+}(B)}$ : $\operatorname{dom} \emptyset_{\Pi_{+}(B)} \rightarrow L^{2}(S)$ is a Fredholm operator and that its index is given by the following formula:

$$
\text { index } D_{\Pi_{+}(B)}=\int \alpha_{0}(x) d x-\frac{1}{2}\left(\eta_{B}(0)+\operatorname{dim} \operatorname{ker} B\right) \text {, }
$$


where $\alpha_{0}$ is the index density which is equal 0 when $\operatorname{dim} X$ is odd, hence $\eta_{B}(s)=\sum_{\lambda \in \operatorname{spec} B, \lambda \neq 0} \operatorname{sign} \lambda|\lambda|^{-s}$ is equal 0 and we are left with the formula

$$
\text { index } D_{\Pi_{+}(B)}=-\operatorname{dim} \operatorname{ker} B_{+} \text {. }
$$

In [17] the second author proves the following equality

$$
\text { index } D_{\Pi_{+}(B)}=\operatorname{index}\left(\Pi_{+}(B) P(\not D)\right) \text {, }
$$

where he considers $\Pi_{+}(B) P(D): H(D) \rightarrow \operatorname{ran} \Pi_{+}(B)$. It follows from [16] that the index of this last operator is equal 0 if and only if $\Pi_{+}(B)$ and $P(D)$ belong to the same connected component of $P \operatorname{diff}_{p^{+}}\left(S_{-} \oplus S_{+}\right)$.

Hitchin [8] showed that $\operatorname{dim} \operatorname{ker} B=2 \operatorname{dim} \operatorname{ker} B_{+}$varies when we deform the metric (the most simple non-trivial examples are all Riemannian surfaces $Y$ with genus $g>3$ ). This means that $\Pi_{+}(B)$ and $P(D)$ change their connected components independently of each other and that under a smooth change of the metric the operator $D_{\Pi_{+}(B)}$ varies in a non-continuous way.

With the self-adjoint case in mind, the second author introduced the subspace $P \operatorname{diff}_{p^{+}}^{G}$ of $P \operatorname{diff}_{p^{+}}$in his paper [18]. It is defined as follows: We fix $P_{0} \in P \operatorname{diff}_{p^{+}}$ and an automorphism $G: L^{2}\left(Y ;\left.E\right|_{Y}\right) \rightarrow L^{2}\left(Y ;\left.E\right|_{Y}\right)$ such that

$$
G^{2}=-I d \quad \text { and } \quad-G P_{0} G=I d-P_{0} .
$$

Such $G$ always exists. Moreover, the space

$$
P \operatorname{diff}_{p^{+}}^{G}:=\left\{P \in P \operatorname{diff}_{p^{+}} \mid-G P G=I d-P\right\}
$$

is also nontrivial from a topological point of view, namely

$$
\pi_{i}\left(P \operatorname{diff}_{p^{+}}^{G}\right) \cong\left\{\begin{array}{ll}
0 & \text { for } i \text { even } \\
\mathbf{Z} & \text { for } i \text { odd }
\end{array},\right.
$$

as was shown in [18]. In the next section we show how to apply this result to the theory of self-adjoint elliptic boundary value problems.

We want to close with some comments concerning applications of the main result of this section. Theorem 3 shows us the possibility of analyzing families of elliptic boundary value problems (for a fixed operator $A$ ) in exactly the same way as was the case with families of elliptic operators over closed manifolds. Later, we will show precisely how-at least in the case of a simple base manifold - the index of such families arises.

\section{The space of Self-Adjoint Elliptic Boundary Value Problems}

Let us now assume that $A$ is formally self-adjoint. In particular this implies the following identities when we consider the representation (1) over the collar

$$
G(t, .) B_{t}=-B_{t}^{*} G(t, \cdot) \text { and } G^{*}(t, .)=-G(t, .) .
$$

In the following we consider a slightly more special case. We assume that all metrics are of product from on the collar $N$ of $Y$ and that $G$ and $B$ do not depend at $t$ over $N$. Moreover, we shall assume that

$$
G^{2}=-I d \text { and } B=B^{*},
$$


which in view of (4) implies that $G B=-B G$. Note that all operators "of Dirac type" satisfy condition (5).

4 Definition. We shall call the realization $A_{R}$ of $A$ a self-adjoint elliptic boundary value problem if and only if

$$
\left(A_{R}\right)^{*}=A_{R} \text {. }
$$

Now in view of Green's formula (and here $A=A^{*}$ )

$$
\langle A u, v\rangle-\langle u, A v\rangle=\int_{Y}\left\langle G\left(\left.u\right|_{Y}\right),\left.v\right|_{Y}\right\rangle,
$$

we have the following fact.

5 Proposition. An element $P \in P \operatorname{diff}_{p^{+}}\left(\left.E\right|_{Y}\right)$ defines an elliptic self-adjoint boundary value problem for $A$ if and only if $I d-P=-G P G$.

Proof. We have $\langle A u, v\rangle-\langle u, A v\rangle=0$ for $u, v \in \operatorname{dom} A_{P}$, hence $\operatorname{ran} G P=\operatorname{ran}(I d-P)$.

The only problem left is that the space $\operatorname{Ell}_{Y}^{*}(A)$ of self-adjoint problems may be empty. To prove that this is not the case let us recall the following result which was proved in [5] and in a different way in [17], namely

$$
P\left(A^{*}\right)=G P(A) G^{-1}
$$

which in our case gives exactly what we want. Hence

6 Proposition. Let $A$ be an elliptic differential formally self-adjoint operator of first order which satisfies (5). Then the realization $A_{P(A)}$ is a self-adjoint elliptic boundary value problem.

As a consequence we obtain the second main result of this paper:

\section{Theorem.}

$$
\pi_{i}\left(\operatorname{Ell}{ }_{Y}^{*}(A)\right) \cong\left\{\begin{array}{ll}
0 & \text { for } i \text { even } \\
\mathbf{Z} & \text { for } i \text { odd }
\end{array} .\right.
$$

In particular the fundamental group of $\operatorname{Ell}_{Y}^{*}(A)$ is $\mathbf{Z}$. This means that the notion of the spectral flow is well defined in this case. We present the simplest non-trivial examples is the next section.

The simplest example is of course provided by the operator $A=-i(d / d x)$ on $[0,2 \pi]$. We take the family $\left\{A_{t}\right\}_{t \in I}$ of realizations of $A$ with the condition $u(1)=e^{2 \pi i t} u(0)$. The eigenvalues of $A_{t}$ are $\{k+t)_{k \in \mathbf{Z}}$ and the corresponding eigenfunctions are $e^{i(k+t) x}$. Moreover, we have $A_{1}=A_{0}$ and we see that there exists a non-trivial spectral flow in this case.

The next example is in dimension 2. Let $\partial$ denote the complex differentiation $\partial / \partial z: C^{\infty}\left(D^{2}\right) \rightarrow C^{\infty}\left(D^{2}\right)$ over the unit disc. We consider the self-adjoint operator

$$
A=\left(\begin{array}{ll}
0 & \bar{\partial} \\
\partial & 0
\end{array}\right)
$$


which takes over $S^{1}$ the following form:

$$
\left(\begin{array}{cc}
0 & -e^{i \phi} \\
e^{-i \phi} & 0
\end{array}\right)\left(\partial_{r}+\left(\begin{array}{cc}
i \partial_{\phi}+1 & 0 \\
0 & -i \partial_{\phi}
\end{array}\right)\right)
$$

Now $H(A)=H(\partial) \oplus H(\bar{\partial})=H_{+} \oplus H_{-}$, where $H_{+}=\left\{\sum_{k \geqq 0} a_{k} z_{k}\right\}$ and $H_{-}=$ $\left\{\sum_{k \leqq 0} a_{k} x_{k}\right\}$. Note that $H_{+} \cap H_{-}=\mathbf{C}$ does not violate (7) because we have a non-trivial $G$ for $\partial$ (and $\bar{\partial}$ ). We obtain

$$
P(A)=\left(\begin{array}{cc}
\left(I d-P_{+}\right)+p_{0} & 0 \\
0 & P_{+}
\end{array}\right),
$$

where $P_{+}$is the projection onto $\operatorname{span}\left\{e^{i k x}\right\}_{k \geqq 0}$ in $L^{2}$ and $p_{0}$ is the projection onto constants. Now we may choose the family $A_{t}$ of realizations of $A$ with the boundary condition $P_{t}=e^{i t \phi} P(A) e^{-i t \phi}$.

In the next dimension we have the first topologically interesting example: the restriction of the signature operator over $S^{3}$ to $D^{3}$. The precise calculations will be published separately.

\section{Spectral Estimates for Self-Adjoint Elliptic Boundary Value Problems}

The main reason for our investigation of the topological structure of the space of elliptic boundary value problems is the search for non-vanishing spectral invariants. We want to transfer an idea of Vafa and Witten [14] (see also Atiyah [1]) to boundary value problems. They use the notion of spectral flow to obtain uniform inequalities for the spectrum of the extended Dirac operator (uniform with respect to the choice of the coefficients bundle) on closed manifolds. Here, we shall show how their global reasoning can be adapted to our context, thus providing both a short-cut alternative and a substantial supplement to the classical (and neo-classical) spectral analysis with its highly subtle derivations of spectral inequalities.

We consider the simplest (and probably the most interesting) case. Let $\operatorname{dim} X=2 k-1$, and let $A$ be a formally self-adjoint operator of Dirac type

$$
A: C^{\infty}(X ; S \otimes \xi) \rightarrow C^{\infty}(X ; S \otimes \xi),
$$

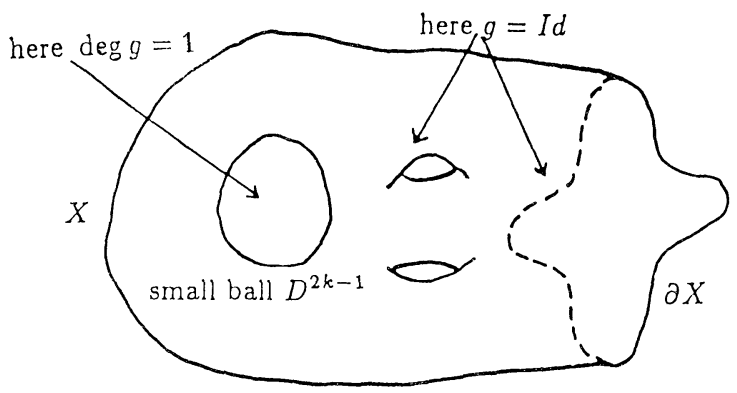

Fig. 1. The choice of $g: X \rightarrow U(N)$ 
where $\xi$ is a Hermitian vector bundle over $X$, and let $\Pi$ be a suitable self-adjoint boundary condition i.e., $\Pi$ is a 0 th order pseudo-differential projection such that $-G \Pi G=I d-\Pi$ (cf. Sect. 2 above).

Now we choose a map $g: X \rightarrow U(N)$ of the form explained in Fig. 1. Since $g$ is equal to $I d$ near the boundary the operator $\Pi \otimes I d_{\mathrm{C}^{N}}=\Pi \oplus \cdots \oplus \Pi$ defines a self-adjoint boundary condition for the operator $A_{1}:=\left(I d \otimes g^{-1}\right) A_{0}(I d \otimes g)$ with $A_{0}:=A \otimes I d_{\mathrm{C}^{N}}$, and in fact for all $A_{t}:=t A_{1}+(1-t) A_{0}, 0 \leqq t \leqq 1$. So we obtain a family of self-adjoint elliptic boundary value problems parametrized over the interval $I$. The family $\left\{\left(A_{t}\right)_{\Pi \otimes I d_{c} N}\right\}_{t \in I}$ of the realizations is a continuous family of self-adjoint unbounded Fredholm operators with discrete spectra and with the periodicity $\operatorname{spec}\left(A_{0}\right)_{\Pi \otimes I d_{\mathrm{C}^{N}}}=\operatorname{spec}\left(A_{1}\right)_{\Pi \otimes I d_{\mathrm{C}^{N}}}$. Because of Theorem 7 there is only one homotopy invariant of such families.

So what is left is to show that the spectral flow of the family of self-adjoint boundary value problems does not vanish.

8 Lemma. Under the previous assumptions we have

$$
\operatorname{sf}\left\{\left(A_{t}\right)_{\Pi \otimes I d C^{N}}\right\}_{t \in I}=\operatorname{rank} \xi .
$$

We postpone the proof of Lemma 8. Its main consequence is the following theorem about the spectral behaviour of the realization $A_{\Pi}$.

9 Theorem. (a) There exists a constant $C$ such that every interval of length $C$ contains an eigenvalue of $A_{\Pi}$.

(b) There exists an upper bound $C n^{1 /(2 k-1)}>\left|\lambda_{n}\right|$ for all eigenvalues of $A_{I}$ indexed by increasing absolute value.

The constants in $(a)$ and $(b)$ depend only on the spin structure of the manifold $X$ and not on the coefficients bundle $\xi$.

Sketch of proof. (a) follows immediately from Lemma 8. In fact, let $\left\{\mathscr{A}_{t}\right\}$ denote the family $\left\{\left(A_{t}\right)_{\Pi \otimes I d_{\mathrm{C}^{N}}}\right\}_{t \in I}$ and let $\left\{t \mapsto j_{r}(t)\right\}_{r \in \mathbf{Z}}$ be a parametrization of the eigenvalues of the family $\left\{\mathscr{A}_{t}\right\}_{t \in I}$. In particular, this means spec $\mathscr{A}_{t}=\left\{j_{r}(t)\right\}_{r \in Z}$. We have spec $\mathscr{A}_{1}=\operatorname{spec} \mathscr{A}_{0}$, and by Lemma 8 we have $j_{r}(1)=j_{r+\operatorname{rank} \xi}(0)$. This actually is the precise meaning of $\operatorname{sf}\left\{\mathscr{A}_{t}\right\}=\operatorname{rank} \xi$.

Since $g=I d$ near the boundary of $X$ we have $A_{1}=A_{0}$ in a collar of the boundary. This makes the difference $A_{1}-A_{0}$ to an $L^{2}$-bounded operator. Hence $\mathscr{A}_{1}-\mathscr{A}_{0}$ is bounded in its domain in $L^{2}\left(X ;(S \otimes \xi) \otimes C^{N}\right)$, and by continuity we have

$$
\left|j_{r}(0)-j_{r}(1)\right| \leqq\left\|\mathscr{A}_{1}-\mathscr{A}_{0}\right\|=: C \text { for all } r \text {, }
$$

hence $\left|j_{r}(0)-j_{r+\operatorname{rank} \xi}(0)\right| \leqq C$. This proves that in any interval of length $C$ there is at least one eigenvalue of $\mathscr{A}_{0}$. The eigenvalues of $\mathscr{A}_{0}$ are the eigenvalues of $A_{\Pi I}$, just with $N$-times multiplicity. Finally, we see that $C$ does not depend on the choice of $\xi$. This proves the uniformity of our estimate.

For the proof of (b) one repeats and sharpens the arguments given above. The main ingredient is the choice of a family $\left\{\gamma_{r}\right\}_{r \in Z}$ of twistings $g_{r}: X \rightarrow U(N)$ such that $\operatorname{ch}\left[X \times \mathbf{C}^{N}, g_{r}\right]=r^{2 k-1} \operatorname{ch}\left[X \times \mathbf{C}^{N}, g_{1}\right]$, where $g_{1}=g$ has "degree" 1 as above.

Remark. Instead of twisting the operator $A$ over the interior of $X$ one could choose a twisting of the boundary condition $\Pi$ or of the boundary component $B$ of the 


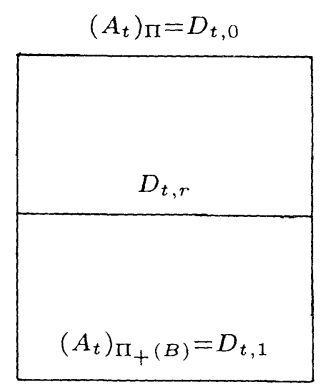

Fig. 2. The family $D_{t, r}$

operator $A$ over the closed manifold $Y$ by means of a topological non-trivial map $g: Y \rightarrow U(N)$. Unfortunately, at present we are not able to give a topological formula for the spectral flow of the families defined in this way.

\section{Remarks Concerning the Proof of Lemma 8}

We sketch the proof of Lemma 8. For simplicity we assume that the "tangential" component $B$ of the operator $A$ is invertible. The necessary generalizations to a non-invertible $B$ may be found in [19], where the second author also discusses the relation of our results to the recent works of Bismut and Cheeger [3, 4], and of Singer [13].

Starting with an arbitrary self-adjoint condition $\Pi$, we obtain a family $\left\{\left(A_{t}\right)_{\Pi}\right\}_{t \in I}$ of self-adjoint unbounded Fredholm operators parametrized over the circle. Since $B$ is invertible, the standard boundary condition $\Pi_{+}(B)$ is self-adjoint. We are going to show that sf $\left\{\left(A_{t}\right)_{\Pi\}_{t \in I}}\right.$ does not depend on the special choice of $\Pi$, in fact sf $\left\{\left(A_{t}\right)_{\Pi}\right\}_{t \in I}=\operatorname{sf}\left\{\left(A_{t}\right)_{\Pi_{+}(B)}\right\}_{t \in I}$. Afterwards, we will prove sf $\left\{\left(A_{t}\right)_{\Pi_{+}(B)}\right\}_{t \in I}=\operatorname{rank} \xi$.

As mentioned above, the space $P \operatorname{diff}_{p^{+}}^{G}$ is path-connected. So, we may choose a path $\left\{\Pi_{r}\right\}_{r \in I}$ in $P \operatorname{diff}_{p^{+}}^{G}$ connecting $\Pi_{0}:=\Pi$ with $\Pi_{1}:=\Pi_{+}(B)$. We consider the family $\left\{D_{t, r}=\left(A_{t}\right)_{\Pi_{r}}\right\}_{t, r \in I}$, (see Fig. 2). Apparently, we have $\operatorname{sf}\left\{S_{t, r}\right\}=\operatorname{sf}\left\{D_{t, r^{\prime}}\right\}$ for any $r, r^{\prime}$, hence $\operatorname{sf}\left\{\left(A_{t}\right)_{\Pi}\right\}=\operatorname{sf}\left\{\left(A_{t}\right)_{\Pi_{+}(B)}\right\}$.

The family $\left\{\left(A_{t}\right)_{\Pi(B)}\right\}_{t \in I}$ consists of standard Atiyah-Singer-Patodi realizations of $A_{t}$. So we can apply results of Cheeger (see [6] and especially [7, p. 209, Remark 6.11], or we may follow the analysis from Atiyah-Patodi-Singer [2]. We obtain that $\eta_{t}(0):=\eta\left(\left(A_{t}\right)_{\Pi_{+}(B)} ; 0\right)$ is well-defined and that $d \eta_{t}(0) / d t$ is local. Because of the fixed boundary condition, the integral which defines $d \eta_{t}(0) / d t$ vanishes near the boundary. Hence it is given by the standard formula on the closed double $\tilde{X}$ of $X$ out of a neighbourhood of $\partial X$.

The detailed calculations can be found in [19].

Acknowledgements. The first author thanks Gerd Grubb for an introduction to the classical (and neoclassical) spectral analysis of elliptic boundary value problems. The second author thanks Jeff Cheeger, Ron Douglas and Werner Müller for valuable discussions concerning the subject of this paper. 


\section{References}

1. Atiyah, M. F.: Eigenvalues of the Dirac operator. In: Hirzebruch, F. et al. (eds.). Arbeitstagung Bonn 1984. Lecture Notes in Mathematics vol. 1111, pp. 251-260. Berlin, Heidelberg, New York: Springer 1985

2. --, Patodi, V. K., Singer, I. M.: Math. Proc. Camb. Phil. Soc. 77, 43-69 (1975)

3. Bismut, J.-M., Cheeger, J.: C. R. Acad Sci. Paris 305, Série I, 127-130 (1987)

4. --,--: Families index for manifolds with boundary, superconnections and cones. (To appear)

5. Booß, B., Wojciechowski, K. P.: Ann. Glob. Analysis and Geometry 4, 349-400 (1988)

6. Cheeger, J.: J. Differential Geom. 18, 575-657 (1983)

7. - - J. Differential Geom. 26, 175-221 (1987)

8. Hitchin, N.: Adv. Math. 14, 1-55 (1974),

9. Palais, R. S. (ed.): Seminar on the Atiyah-Singer Index Theorem. Ann. of Math. Studies vol. 57. Princeton, NJ: Princeton University Press 1965

10. Pressley, A., Segal, G. B.: Loop Groups. Oxford: Oxford University Press 1986

11. Seeley, R. T.: Topics in pseudo-differential operators. In: CIME Conference on Pseudo-Differential Operators (Stresa 1968). pp. 167-305 Rome: (ed.) Cremonese 1969

12. Segel, G. B., Wilson, G.: Pub. Math. I.H.E.S. 61, 5-65 (1985)

13. Singer, I. M.: The $\eta$-invariant and the index (to appear)

14. Vafa, C, Witten, C.: Commun. Math. Phys. 95, 257-274 (1984)

15. Witten, E.: Commun. Math. Phys. 113, 529-600 (1988)

16. Wojciechowski, K. P.: J. Operator Theory 15, 207-216 (1986)

17. --: J. Operator Theory 20, 107-115 (1988)

18. - : The homotopy groups of the space of Pseudodifferential projections with the same principal symbol. Preprint 1987

19. - : Spectral flow of families of elliptic boundary value problems and adiabatic limits of the $\eta$-invariant. Preprint 1988

Communicated by A. Jaffe

Received January 29, 1988; in revised form September 13, 1988 
